\title{
Periostin on the road to nonalcoholic fatty liver disease
}

\author{
Stergios A. Polyzos ${ }^{1}$ (1) Athanasios D. Anastasilakis ${ }^{2}$
}

Received: 27 October 2015/Accepted: 6 November 2015/Published online: 19 November 2015

(C) Springer Science+Business Media New York 2015

\section{Introduction}

Nonalcoholic fatty liver disease (NAFLD) is a global public health issue, affecting approximately one-third of the general population. NAFLD ranges from nonalcoholic simple steatosis (SS) to nonalcoholic steatohepatitis (NASH), characterized by inflammation and/or fibrosis, NASH-related cirrhosis and hepatocellular carcinoma [1]. Liver biopsy remains the gold standard in the diagnosis of NAFLD, although identification of noninvasive biomarkers is a field of intense research [2]. Even more, despite its high prevalence, the treatment of NAFLD currently remains an unmet medical need [3].

Periostin (encoded by POSTN), a member of the vitamin-K-dependent $\gamma$-carboxylated protein family, is a secreted extracellular matrix protein expressed in collagenrich connective tissues and involved in cell recruitment and adhesion [4]. Its name (derived from the Greek words "peri" and "ostoun", meaning around the bone) refers to its original identification in periosteal osteocytes and osteoblasts. The role of periostin in the human skeleton is yet unclear; it has been positively associated with fracture risk [5], but not with bone mineral density [5, 6], thereby implying that periostin is primarily associated with the organic rather than the mineral component of the skeleton. Beyond the bone, periostin exerts a multifunctional facet,

Stergios A. Polyzos

stergios@endo.gr

1 Second Medical Clinic, Ippokration General Hospital, Aristotle University of Thessaloniki, 13 Simou Lianidi, 55134 Thessaloniki, Macedonia, Greece

2 Department of Endocrinology, 424 General Military Hospital, Thessaloniki, Greece being linked with several inflammatory diseases, including asthma, skin inflammation, and atherosclerosis, but also malignant diseases, including breast, colon, head and neck, pancreatic, lung, papillary thyroid, ovarian, gastric and hepatocellular carcinoma, and cholangiocarcinoma [4].

\section{Periostin in hepatic metabolism}

There is experimental evidence implicating periostin in hepatic steatosis, inflammation, and fibrosis. A eightfold higher hepatic periostin expression was shown in mice fed a high-fat diet compared with a chow diet [7]. Hepatic periostin was also markedly higher in $o b / o b$ and $d b / d b$ mice, known models of monogenic obesity and NAFLD, compared to lean controls [7]. Furthermore, overexpression of periostin in the liver of wild-type mice promoted hypertriglyceridemia and hepatic steatosis, possibly through reducing the expression of peroxisome proliferator-activated receptor (PPAR)- $\alpha$ pathway, thereby reducing fatty acid oxidation [7]. On the other hand, knockdown of periostin gene or administration of a periostin-neutralizing antibody in $d b / d b$ mice reduced hepatic and serum triglycerides and improved steatosis, without affecting body weight, fat mass or transaminase levels [7].

Other authors showed that periostin is highly upregulated in methionine-choline deficient (MCD) diet-fed mice, a model known to develop NASH, compared with mice on a chow diet [8]. In these mice, hepatic periostin was mainly distributed around steatotic hepatocytes. Notably, periostin knockout mice on a MCD diet had a markedly lower degree of hepatic steatosis, inflammation and fibrosis compared with wild-type mice also on MCD diet [8].

Similarly, periostin expression was upregulated in mice treated with carbon tetrachloride-induced acute and chronic 
liver fibrosis, whereas liver fibrosis was abrogated in periostin knockout mice [9]. Carbon tetrachloride treatment significantly increased a-smooth muscle actin, fibronectin, and type I collagen levels in wild-type mice, which remained unaffected in periostin knockout mice. Notably, periostin-deficient mice showed lower levels of transforming growth factor (TGF)- $\beta 1$ and TGF- $\beta 2$ (factors linked to hepatic fibrosis) compared with wild-type mice after carbon tetrachloride treatment [9]. Furthermore, mRNA levels of proinflammatory and profibrotic factors, including chemokine ligand 2 , interleukin (IL)-6, IL- $1 \beta$, tumor necrosis factor (TNF)- $\alpha$, and tissue inhibitor of metalloproteinase-1 were significantly lower in periostindeficient mice than in wild-type mice after carbon tetrachloride treatment [9].

Importantly, in vitro activation of human hepatoblastoma cells (HepG2) with the proinflammatory and profibrogenic cytokines TNF- $\alpha$, IL-17, or both, enhanced the expression of periostin by 3.5-, 4.4-, or 13.3-fold, respectively, compared to untreated cells [10]. It seems that periostin may mediate the type I collagen disposition induced by TNF- $\alpha$ and IL-6, thereby resulting in fibrosis [10].

There are limited data for periostin in human NAFLD. In line with experimental models, hepatic periostin expression and serum periostin levels were increased in NAFLD patients compared with controls [7]. Other authors also reported higher serum periostin levels in patients with acute or chronic hepatitis compared with controls; in the same study, periostin levels were well associated with TGF- $\beta 1$ and TGF- $\beta 2$ [9]. Taken together, the aforementioned experimental and human data [7-10], periostin may mediate hepatic steatosis, inflammation, and fibrosis.

\section{What is new in "Endocrine"}

As a rational consequence of the previous studies, Zhu et al. performed a case-control study of 86 ultrasoundproven NAFLD patients and 86 controls to evaluate periostin as a noninvasive biomarker of NAFLD [11]. They observed that serum periostin levels were higher in NAFLD patients than controls, and that the frequency of NAFLD was escalading from the first to the third tertile of periostin, being approximately $30 \%$ (first tertile), $53 \%$ (second tertile), and $67 \%$ (third tertile). More importantly, periostin levels were associated with the presence of NAFLD independently from potential cofounders, including adiposity (body mass index and waist to hip ratio), which is a significant determinant of NAFLD [11]. Moreover, Zhu et al. evaluated a receiver operating characteristic (ROC) curve, according to which periostin levels were shown to predict NAFLD, albeit with relatively moderate sensitivity and specificity [11]. Finally, periostin was positively correlated with other factors contributing to inflammation in NAFLD, including lipocalin-2, hepatic growth factor, resistin, IL-6, and IL-8, whereas negatively with adiponectin, an insulin-sensitizing and anti-inflammatory adipokine [11].

\section{Future directions}

Following the Zhu et al. publication [11], the rational next steps of periostin on the road to NAFLD may be the comparative evaluation of its circulating levels in histologically confirmed patients with SS and NASH. Since periostin was experimentally linked to inflammation and fibrosis, higher periostin levels are expected in NASH than SS patients. If this were proved, then the accuracy of circulating periostin, alone or combined with other noninvasive factors, to predict NASH and/or hepatic fibrosis, the main prognostic factor for advanced disease, would be of importance. Subsequently, a long-term cohort study would be the ideal way to validate the accuracy of periostin as a noninvasive biomarker for NASH and/or hepatic fibrosis. It should be highlighted that high periostin levels were shown to be associated with poor prognosis (overall and relapsefree survival) in a cohort of patients with hepatocellular carcinoma (albeit mostly related to hepatitis B) [12].

The role of periostin in NAFLD becomes of higher importance considering the existence of periostin antagonists, aptamers, which are modified nucleic acids that specifically bind periostin and inhibit its function. These aptamers seem to block the interaction between periostin and its cell surface receptor, thereby impairing the activation of periostin signaling pathways. Periostin antagonists have currently been investigated in breast [13] and gastric [14] cancer; however, a wider understanding of the role and mechanisms of periostin in hepatic inflammation and fibrosis may render periostin antagonists a rational therapeutic alternative for NASH treatment.

\section{Compliance with ethical standards}

Conflict of interest The authors declare that they have no conflict of interest.

\section{References}

1. S.A. Polyzos, J. Kountouras, C. Zavos, Nonalcoholic fatty liver disease: the pathogenetic roles of insulin resistance and adipocytokines. Curr. Mol. Med. 9, 299-314 (2009)

2. S.A. Polyzos, C.S. Mantzoros, Necessity for timely noninvasive diagnosis of nonalcoholic fatty liver disease. Metabolism 63, 161-167 (2014)

3. S.A. Polyzos, J. Kountouras, C. Zavos, G. Deretzi, Nonalcoholic fatty liver disease: multimodal treatment options for a 
pathogenetically multiple-hit disease. J. Clin. Gastroenterol. 46, 272-284 (2012)

4. A.Y. Liu, H. Zheng, G. Ouyang, Periostin, a multifunctional matricellular protein in inflammatory and tumor microenvironments. Matrix Biol. 37, 150-156 (2014)

5. J.C. Rousseau, E. Sornay-Rendu, C. Bertholon, R. Chapurlat, P. Garnero, Serum periostin is associated with fracture risk in postmenopausal women: a 7-year prospective analysis of the OFELY study. J. Clin. Endocrinol. Metab. 99, 2533-2539 (2014)

6. A.D. Anastasilakis, S.A. Polyzos, P. Makras, M. Savvides, G.T. Sakellariou, A. Gkiomisi, A. Papatheodorou, E. Terpos, Circulating periostin levels do not differ between postmenopausal women with normal and low bone mass and are not affected by zoledronic acid treatment. Horm. Metab. Res. 46, 145-149 (2014)

7. Y. Lu, X. Liu, Y. Jiao, X. Xiong, E. Wang, X. Wang, Z. Zhang, H. Zhang, L. Pan, Y. Guan, D. Cai, G. Ning, X. Li, Periostin promotes liver steatosis and hypertriglyceridemia through downregulation of PPAR $\alpha$. J. Clin. Invest. 124, 3501-3513 (2014)

8. Y. Li, S. Wu, S. Xiong, G. Ouyang, Deficiency of periostin protects mice against methionine-choline-deficient diet-induced non-alcoholic steatohepatitis. J. Hepatol. 62, 495-497 (2015)

9. Y. Huang, W. Liu, H. Xiao, A. Maitikabili, Q. Lin, T. Wu, Z. Huang, F. Liu, Q. Luo, G. Ouyang, Matricellular protein periostin contributes to hepatic inflammation and fibrosis. Am. J. Pathol. 185, 786-797 (2015)

10. S. Amara, K. Lopez, B. Banan, S.K. Brown, M. Whalen, E. Myles, M.T. Ivy, T. Johnson, K.L. Schey, V. Tiriveedhi, Synergistic effect of pro-inflammatory $\mathrm{TNF} \alpha$ and IL-17 in periostin mediated collagen deposition: potential role in liver fibrosis. Mol. Immunol. 64, 26-35 (2015)

11. J.Z. Zhu, H.T. Zhu, Y.N. Dai, C.X. Li, Z.Y. Fang, D.J. Zhao, X.Y. Wan, Y.M. Wang, F. Wang, C.H. Yu, Y.M. Li, Serum periostin is a potential biomarker for non-alcoholic fatty liver disease: a case-control study. Endocrine (2015). doi:10.1007/ s12020-015-0735-2

12. Y. Lv, W. Wang, W.D. Jia, Q.K. Sun, M. Huang, H.C. Zhou, H.H. Xia, W.B. Liu, H. Chen, S.N. Sun, G.L. Xu, High preoperative levels of serum periostin are associated with poor prognosis in patients with hepatocellular carcinoma after hepatectomy. Eur. J. Surg. Oncol. 39, 1129-1135 (2013)

13. Y.J. Lee, I.S. Kim, S.A. Park, Y. Kim, J.E. Lee, D.Y. Noh, K.T. Kim, S.H. Ryu, P.G. Suh, Periostin-binding DNA aptamer inhibits breast cancer growth and metastasis. Mol. Ther. 21, 1004-1013 (2013)

14. G.X. Liu, H.Q. Xi, X.Y. Sun, B. Wei, Role of periostin and its antagonist PNDA-3 in gastric cancer metastasis. World J. Gastroenterol. 21, 2605-2613 (2015) 